\title{
Laser spectroscopy of three-body antiprotonic and pionic helium atoms at CERN and PSI
}

\section{Masaki Hori $^{a, b, *}$}

${ }^{a}$ Max-Planck-Institut für Quantenoptik,

Hans-Kopfermann-Strasse 1, D-85748 Garching, Germany

${ }^{b}$ Fakultät für Physik, Ludwig-Maximilians-Universität München, D-80799 Munich, Germany

E-mail: Masaki.Hori@cern.ch

Some recent results on laser spectroscopy experiments of three-body antiprotonic helium atoms carried out at the Antiproton Decelerator facility of CERN, and pionic helium atoms carried out at the $590 \mathrm{MeV}$ ring cyclotron facility of the Paul Scherrer Institute are reviewed. An infrared laser transition $(n, \ell)=(17,16) \rightarrow(17,15)$ of pionic helium at a frequency of $v \approx 183760 \mathrm{GHz}$ was detected.

*** Particles and Nuclei International Conference - PANIC2021 ***

*** 5 - 10 September, $2021 * * *$

$* * *$ Online $* * *$

${ }^{*}$ Speaker 
The ASACUSA collaboration has measured the transition frequencies of antiprotonic helium atoms $\left(\bar{p}^{4} \mathrm{He}^{+} \equiv \bar{p}+{ }^{4} \mathrm{He}^{2+}+e^{-}\right.$and $\left.\bar{p}^{3} \mathrm{He}^{+}\right)$using the Antiproton Decelerator (AD) [1-8]. This three-body atom consists of a helium nucleus, an electron in the ground state, and an antiproton that occupies a state with large principal and orbital angular momentum quantum numbers of $n \approx \ell+1 \approx 38$ [9]. The atoms are produced by allowing antiprotons to come to rest in helium targets. Since the electromagnetic cascade processes such as Auger decay [10] are suppressed, the atom retains a microsecond-scale lifetime against antiproton annihilation in the helium nucleus. By utilizing sub-Doppler two-photon laser spectroscopy [6, 11] or buffer gas cooling [7], 16 transition frequencies were measured to fractional precision of 2.3-16 parts in 109. By comparing these frequencies with the results of quantum electrodynamics (QED) calculations [1], the antiprotonto-electron mass ratio was determined as $M_{\bar{p}} / m_{e}=1836.1526734(15)$ [7]. These studies are complementary to those on $\mathrm{HD}^{+}$ions $[12,13]$. The results were used to set upper limits on fifth forces between antiprotons and nucleons at atomic length scales [14-16], and on forces that may arise between an electron and antiproton mediated by hypothetical spin=0 or 1 bosons [17].

The PiHe collaboration at PSI recently carried out laser spectroscopy of the analogous pionic helium atom $\left(\pi^{4} \mathrm{He}^{+} \equiv \pi^{-}+{ }^{4} \mathrm{He}^{2+}+e^{-}\right)[18-22]$. In this three-body system, a negatively-charged pion occupies a highly excited state with $n \approx \ell+1 \approx 17$. The atom has a much shorter, nanosecondscale lifetime against $\pi^{-}$absorption in the nucleus which makes the laser spectroscopy measurements more difficult compared to the $\bar{p} \mathrm{He}^{+}$case. The $\pi^{-}$mass may in principle be precisely determined from the transition frequencies of $\pi^{4} \mathrm{He}^{+}$in a similar way to $\bar{p} \mathrm{He}^{+}$[23-25] to $\mathrm{eV}$-scale precision or better. This may help set upper limits on the laboratory constraints on the muon antineutrino mass which would then be limited by the experimental uncertainty of the momentum of the $\mu^{+}$ emerging from $\pi^{+}$decay [26]. Upper limits may also be set on exotic forces between the $\pi^{-}$and helium nuclei. The $\pi^{4} \mathrm{He}^{+}$atom is interesting as it has no hyperfine structure due to the spin-spin interaction between the spin $=0$ alpha particle and the pion. QED effects in this boson-boson bound state may be precisely studied by these experiments. In a recent experiment [22], $800 \mathrm{ps-long}$ laser pulses of wavelength $\lambda \approx 1631 \mathrm{~nm}$ excited a transition from the pionic state $(n, \ell)=(17,16)$ that had a nanosecond-scale lifetime, to a state $(17,15)$ with a $\tau=5$ ps Auger lifetime [21]. This lead to immediate pion absorption and breakup of the helium nucleus. The laser resonance was then revealed as a peak in the measured rates of the resulting neutrons, protons, and deuterons.

The PSI cyclotron facility provided a $\pi^{-}$beam of momentum $p=83-87 \mathrm{MeV} / \mathrm{c}$ and intensity $N_{\pi}=(2-3) \times 10^{7} \mathrm{~s}^{-1}$. The $\pi^{-}$traversed a segmented plastic scintillator plate before entering the helium target (Fig. 1 (a)). The $\pi \mathrm{He}^{+}$were thus produced at a rate $>3 \times 10^{5} \mathrm{~s}^{-1}$. The $\pi^{-}$arrived at intervals of $\Delta t=19.75 \mathrm{~ns}$ which arose from the $f_{a}=50.63 \mathrm{MHz}$ accelerating radiofrequency of the cyclotron. The interrogating laser pulses of energy $E=10 \mathrm{~mJ}$ and repetition rate of $f_{r}=80.1 \mathrm{~Hz}$ were generated by an injection-seeded, optical parameteric generator (OPG) and amplifier (OPA) (Fig. 1 (b)). The laser beam irradiated the atoms at a time $t=9 \mathrm{~ns}$ after $\pi^{-}$arrival, with an estimated probability of coincidence of $\approx 10^{-3}$ [22]. The arrival times of the neutrons, protons, and deuterons of $\mathrm{MeV}$-scale kinetic energies at a scintillation counter array that surrounded the target were then measured. For this the analog waveform of the counter signals were recorded [27-30]. An earlier variant of the electronics was used to measure the limits on the cross sections of antiprotons of energy $E \approx 125 \mathrm{keV}$ annihilating in some target foils [29, 31, 32]. The results were compared with measurements of the annihilation cross sections of $E=5.3 \mathrm{MeV}$ antiprotons [30, 33, 34]. 


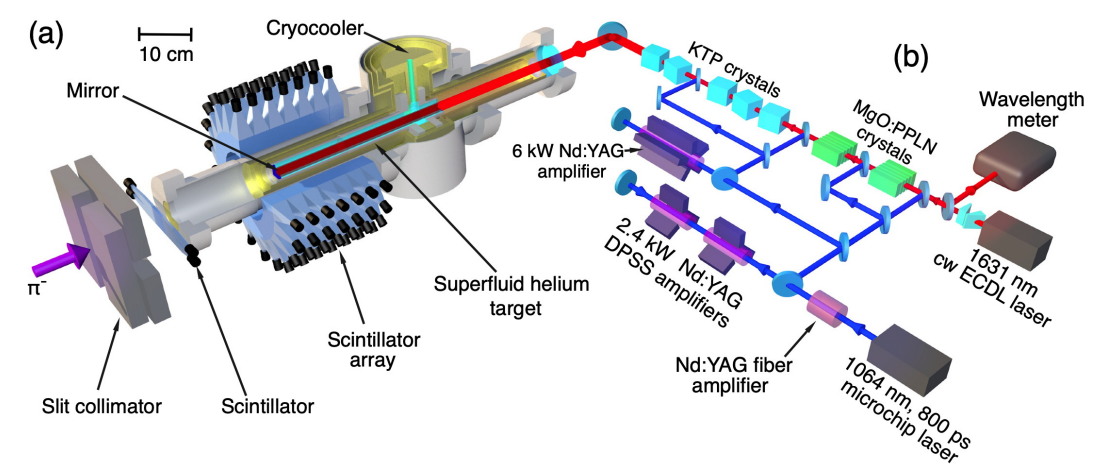

Figure 1: (a): Layout of the experimental target, and (b): the laser system. From Ref. [22].

Fig. 2 (a) (blue histogram) shows the time spectrum of particle arrivals at the scintillator array which were measured without the laser irradiation. The peaks at time $t=0$ and $19.75 \mathrm{~ns}$ arise from pion arrivals at the helium target that underwent prompt $\pi^{-}$absorption. On the other hand, a $(2.1 \pm 0.7) \%$ fraction of the $\pi^{-}$constituted the time spectrum of $\pi^{4} \mathrm{He}^{+}$that decayed with a lifetime of $\tau=(7 \pm 2) \mathrm{ns}$ in the intervals between the $\pi^{-}$arrivals. The time spectrum indicated by filled circles which is superimposed in Fig. 2 (a) was measured with the laser tuned to a wavelength $\lambda \approx 1631.4 \mathrm{~nm}$ corresponding to the transition $(17,16) \rightarrow(17,15)$. The signal of the laser resonance was detected at time $t=9 \mathrm{~ns}$. The detection rate of $3 \mathrm{~h}^{-1}$ resonant events is compatible with the results of Monte Carlo simulations [21] in which most of the long-lived atoms are assumed to populate state $(17,16)$. The signal disappeared when the laser was detuned off the resonance.

The spectrum of Fig. 2 (b) was obtained by scanning the laser frequency and plotting the number of resonant events. The statistical uncertainty is indicated by vertical error bars. The resonance width primarily arises from the Auger width $\Gamma_{A}=33 \mathrm{GHz}$ of the daughter state $(17,15)$ [21], collisional [19] and power broadening, and the laser linewidth. Collisions with helium atoms may shorten $[18,35]$ the state lifetimes and cause more broadening. The best fit (blue curve) of two overlapping Lorentzian functions that take the fine structure sublines arising from the interaction between the electron's spin and orbital angular momentum is shown superimposed. The resonance centroid was determined as $v_{\exp }=183760(6)(6) \mathrm{GHz}$ with a statistical uncertainty of $6 \mathrm{GHz}$. The systematic uncertainty of $6 \mathrm{GHz}$ arises from contributions related to the selection of the fit function and the laser frequency. The experimental frequency is larger than the theoretical one $v_{\text {th }}=(183681.8 \pm 0.5) \mathrm{GHz}$ [21] which is believed to be due to atomic collisions [18, 19, 35, 36]. A frequency shift $\Delta v=96-142 \mathrm{GHz}$ was predicted from the impact approximation of the binary collision theory of spectral lineshapes [19]; this roughly agreed with the experimental results.

Previous experiments on $\bar{p} \mathrm{He}^{+}[6,7,37]$ were based on a radiofrequency quadrupole decelerator which slowed down the $E=5.3 \mathrm{MeV}$ beam of the AD to an energy $E<100 \mathrm{keV}$ [38]. The new ELENA facility [39] provides an electron-cooled beam of $E=100 \mathrm{keV}$. The ASACUSA collaboration provided the beam profile monitors [40] of the beamlines that transport the antiprotons. This higher-quality beam will allow us to carry out two-photon laser spectroscopy of some $\bar{p} \mathrm{He}^{+}$ transitions with natural widths that are two orders of magnitude smaller than those that were previously measured [11]. Improved lasers will be used [41]. We will also study antiprotonic helium ions [42-44] that consist of a helium nucleus and antiproton occupying the $n \approx 30$ states. At PSI we plan to search for the $\pi^{4} \mathrm{He}^{+}$transitions in the region $n \approx 16$ which calculations predict are narrower by a factor of at least $10^{-3}$ compared to the observed one [21]. The precision of the 

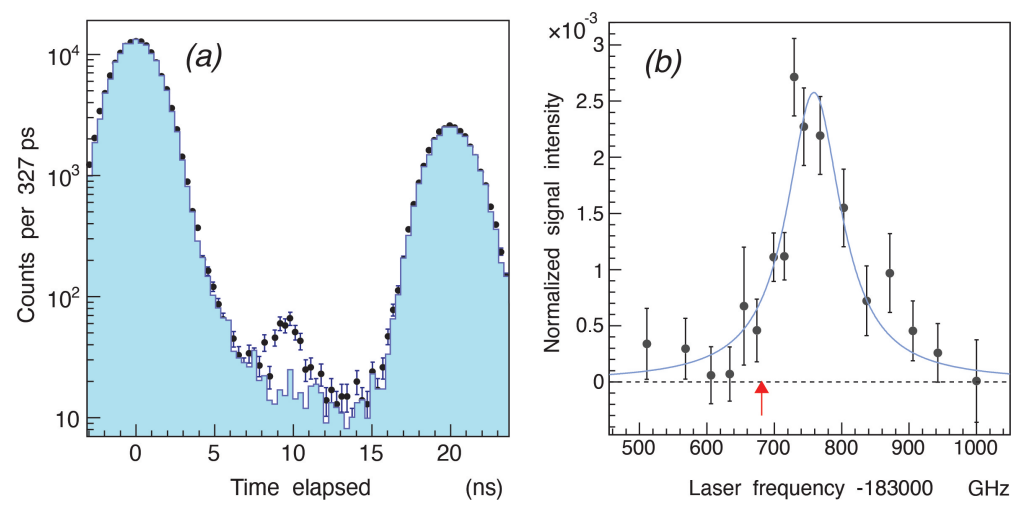

Figure 2: (a): Time spectra of nuclear fragments measured with (filled circles) and without (blue histogram) laser irradiation. The peak in the former spectrum at $t=9 \mathrm{~ns}$ corresponds to the resonance signal. (b): The spectral profile with the theoretical transition frequency indicated by the red arrow. From Ref. [22].

theoretical frequencies is now limited by the experimental uncertainty of the $\pi^{-}$mass, whereas the relative precision of the calculations [21] can be improved to better than $10^{-8}$.

This work was supported by the Max-Planck-Gesellshaft and the European Research Council.

\section{References}

[1] V.I. Korobov, L. Hilico and J.-P. Karr, Phys. Rev. Lett. 112 (2014) 103003.

[2] M.-H. Hu, et al., Chem. Phys. Lett. 654 (2016) 114.

[3] M. Hori et al., Phys. Rev. Lett. 96 (2006) 243401.

[4] T. Kobayashi et al., J. Phys. B 46 (2013) 245004.

[5] S. Friedreich et al., J. Phys. B 46 (2013) 125003.

[6] M. Hori et al., Nature 475 (2011) 484.

[7] M. Hori et al., Science 354 (2016) 610.

[8] A. Sótér, H. Aghai-Khozani, D. Barna, A. Dax, L. Venturelli, M. Hori, Nature 603 (2022) 411.

[9] M. Hori et al., Phys. Rev. Lett. 89 (2002) 093401.

[10] H. Yamaguchi et al., Phys. Rev. A 66 (2002) 022504.

[11] M. Hori and V.I. Korobov, Phys. Rev. A 81 (2010) 062508.

[12] S. Alighanbari et al., Nature 581 (2020) 152.

[13] S. Patra et al., Science 369 (2020) 1238.

[14] E. Salumbides, W. Ubachs and V.I. Korobov, J. Mol. Spectrosc. 300 (2014) 65.

[15] J. Murata and S. Tanaka, Class. Quantum Gravity 32 (2015) 033001. 
[16] M. Germann et al., Phys. Rev. Research 3 (2021) L022028.

[17] F. Ficek et al., Phys. Rev. Lett. 120 (2018) 183002.

[18] V.I. Korobov et al., J. Phys. B 48 (2015) 245006.

[19] B. Obreshkov and D. Bakalov, Phys. Rev. A 93 (2016) 062505.

[20] D. Baye and J. Dohet-Eraly, Phys. Rev. A 103 (2021) 022823.

[21] M. Hori, A. Sótér and V.I. Korobov, Phys. Rev. A 89 (2014) 042515.

[22] M. Hori, H. Aghai-Khozani, A. Sótér, A. Dax and D. Barna, Nature 581 (2020) 37.

[23] S. Lenz et al., Phys. Lett. B 416 (1998) 50.

[24] M. Trassinelli et al., Phys. Lett. B 759 (2016) 583.

[25] M. Daum, R. Frosch and P.-R. Kettle, Phys. Lett. B 796 (2019) 11.

[26] K. Assamagan et al., Phys. Rev. D 53 (1996) 6065.

[27] M. Hori, K. Yamashita, R. Hayano and T. Yamazaki, Nucl. Instr. and Meth. A 496 (2003) 102.

[28] A. Sótér et al., Rev. Sci. Instrum. 85 (2014) 023302.

[29] K. Todoroki et al., Nucl. Instr. and Meth. A 835 (2016) 110.

[30] Y. Murakami, H. Aghai-Khozani and M. Hori, Nucl. Instrum. and Meth. A 933 (2019) 75.

[31] H. Aghai-Khozani et al., Eur. Phys. J. Plus 127 (2012) 125.

[32] H. Aghai-Khozani et al., Nucl. Phys. A 1009 (2021) 122170.

[33] A. Bianconi et al., Phys. Lett. B 704 (2011) 461.

[34] H. Aghai-Khozani et al., Nucl. Phys. A 970 (2018) 366.

[35] M. Hori et al., Phys. Rev. Lett. 87 (2001) 093401.

[36] D. Bakalov et al., Phys. Rev. Lett. 84 (2000) 2350.

[37] M. Hori and J. Walz, Prog. Part. Nucl. Phys. 72 (2013) 206.

[38] M. Hori et al., Phys. Rev. Lett. 91 (2003) 123401.

[39] D. Gamba et al., ELENA commissioning, in Proceedings of North American Particle Accelerator Conference NAPAC2019, Lansing MI, USA, p. WEYBB1, 2019.

[40] M. Hori, Rev. Sci. Instrum. 76 (2005) 113303.

[41] M. Hori and A. Dax, Opt. Lett. 34 (2009) 1273. 
[42] M. Hori et al., Phys. Rev. Lett. 94 (2005) 063401.

[43] J. Zatorski and K. Pachucki, Phys. Rev. A 82 (2010) 052520.

[44] G.Y. Korenman and S.N. Yudin, Eur. Phys. J. D 75 (2021) 64. 